\title{
TILs in rabbit mammary carcinomas
}

\author{
Contributor: , Sandra Schöniger ${ }^{1}$ \\ 1, Targos Molecular Pathology GmbH, Germaniastrasse 7, 34119 Kassel, \\ Germany; sschoeniger@yahoo.com
}

Version received: 1 September 2020

check for

updates

\section{Definition}

Tumor infiltrating lymphocytes (TILs) are key components of the tumor microenvironment that mediate the anti-tumor immune response. In breast cancer of humans, TILs represent prognostic and predictive biomarkers. For their standardized evaluation in routinely (hematoxylin and eosin) stained tissue sections, international guidelines exist. Recently, TILs have also been analyzed in pet rabbit mammary carcinomas according to these international guidelines. Results of the study on rabbit mammary carcinomas showed a statistically significant association between higher TIL numbers in stromal TIL hotspot areas and microscopic parameters indicative of a better tumor differentiation, i.e. decreased mitotic count, lower histological tumor grade and higher percentage of calponin positive tumor cells. These findings suggest that in rabbit mammary carcinomas TIL hotspot areas may exert an influence on the biological behavior of these tumors. The present study contributes to comparative pathology. In addition, it provides the basis for further investigations into the impact of TILs on clinical parameters of pet rabbit mammary carcinomas.

\section{Introduction}

Nowadays, rabbits are popular pets ${ }^{[\underline{1}]}$ and mammary carcinomas are quite commonly diagnosed in does. ${ }^{[2][\underline{3}]}$ In women, breast carcinoma is worldwide the most frequent cancer type. $[\underline{4}][\underline{5}][\underline{6}]$

Studies revealed comparative features of mammary tumors of pet rabbits and breast cancer in humans,

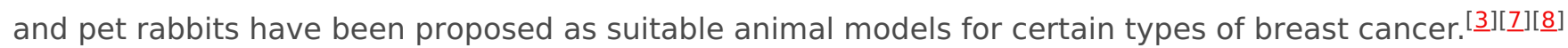

Majority of breast cancer cases in humans ${ }^{[\underline{4}]}$ and mammary carcinomas in pet rabbits ${ }^{[\underline{7}][\underline{8}][\underline{9}][\underline{10}]}$ are histologically diagnosed as invasive adenocarcinomas. The histological grading scheme of Elston and Ellis ${ }^{[11]}$ is used to determine the differentiation of mammary carcinomas in humans ${ }^{[12]}$ as well as in

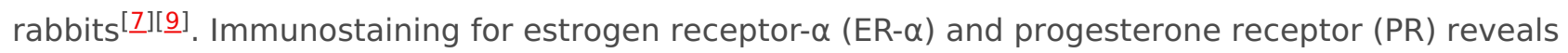
expression of these hormone receptors in molecular subtypes of human[4][12] and in rabbit mammary carcinomas. ${ }^{[7]}$ The disease affects most frequently middle aged to older individuals: In women, $80 \%$ of breast cancer cases are diagnosed at an age $\geq 50$ years, $[\underline{5}][\underline{13}]$ and the average age of does with mammary carcinomas is 4.9-5.5 years. $[\underline{\underline{z}}][\underline{9}][\underline{10}]$ The reproductive age of female rabbits starts with 4-6 months, and the life expectancy of pet rabbits is 6-13 years. ${ }^{[14]}$ In rabbits, elevated serum prolactin concentrations are associated with benign proliferative mammary gland lesions,,$\underline{15}][\underline{16]}][\underline{17]}$ that may undergo neoplastic transformation in rare cases. ${ }^{[17]}$ In women a rise in prolactin for a longer duration represents a predisposing factor for breast cancer development. ${ }^{[18]}$ Investigations into mammary tumor development in breeding colonies of rabbits showed clustering in distinct families suggesting a genetic predisposition. ${ }^{[19]}$ In humans, familial breast cancer accounts for approx. 7\% of all breast cancer cases, ${ }^{[20]}$ and it is in $25 \%$ attributed to mutations in breast cancer gene 1 and 2 (BRCA 1; BRCA 2). [6]

Close similarities between the immune system of rabbits and humans $[\underline{21]}[\underline{22}]$ facilitate the use of the

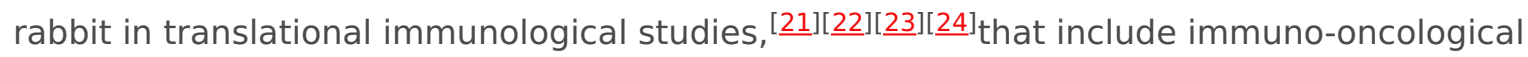


research. $[\underline{25}][\underline{26}]$

The fate of a tumor (regression, progression, metastases) is markedly influenced by the hosts immune response that include components of the innate and adaptive $[\underline{25]}[\underline{27}]$ of tumor infiltrating lymphocytes

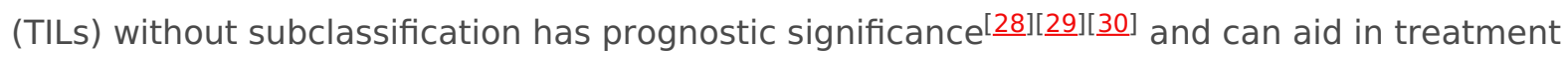

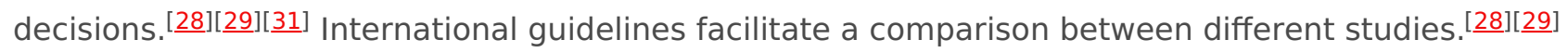

\section{Tumor infiltrating lymphocytes in pet rabbit mammary carcinomas}

The study of Schöniger et al. ${ }^{[32]}$ examined TILs in hematoxylin and eosin stained sections of 107 pet rabbit mammary carcinomas according to guidelines established for breast cancer in humans. [208][29]

TIL evaluation encompassed stromal lymphocytes and plasma cells, ${ }^{[32]}$ whereas stromal TILs are defined as stromal area occupied by TILs related to the evaluated stromal area of a tumor. ${ }^{[28][29]}$ TILs were examined using the $20 x$ objective on a Zeiss microscope A1. ${ }^{[32]}$ For each case, the average percentage of TILs over the entire stromal area as well as the maximal and minimal number of TILs per 20x objective field of view (FOV) were determined. ${ }^{[32]}$ Analyses were performed separately for the central tumor (CT) area and the invasive margin (IM). [32] The latter is defined as $1 \mathrm{~mm}$ wide zone centered on the outermost margin of the tumor cells. ${ }^{[29]}$ Results from the TIL evaluation were statistically correlated with those histological and immunohistochemical findings that may influence the biological behavior of mammary carcinomas, i.e. proliferative activity, tumor differentiation, ER $\alpha$ and PR status as well as calponin expression in tumor cells; ${ }^{[32]}$ these data were obtained from the diagnoatic records. ${ }^{[7]}[\underline{8}]$ For evaluation of the proliferative activity, tumor cells with mitoses were counted in ten 40x objective FOVs. $\left.{ }^{[7]}\right]$ To examine the degree of tumor differentiation, the histological tumor grade was determined ${ }^{[\underline{7}]}$ using the 3-tier grading system of Elston and Ellis. ${ }^{[11]}$ The ER $\alpha$ and PR status and calponin expression were assessed by immunohistochemistry. [] $]$

Results showed that the majority of pet rabbit mammary carcinomas contained 1-10\% average stromal TILs in the CT and at the IM (Figure 1). $\underline{\text { [32] }}$

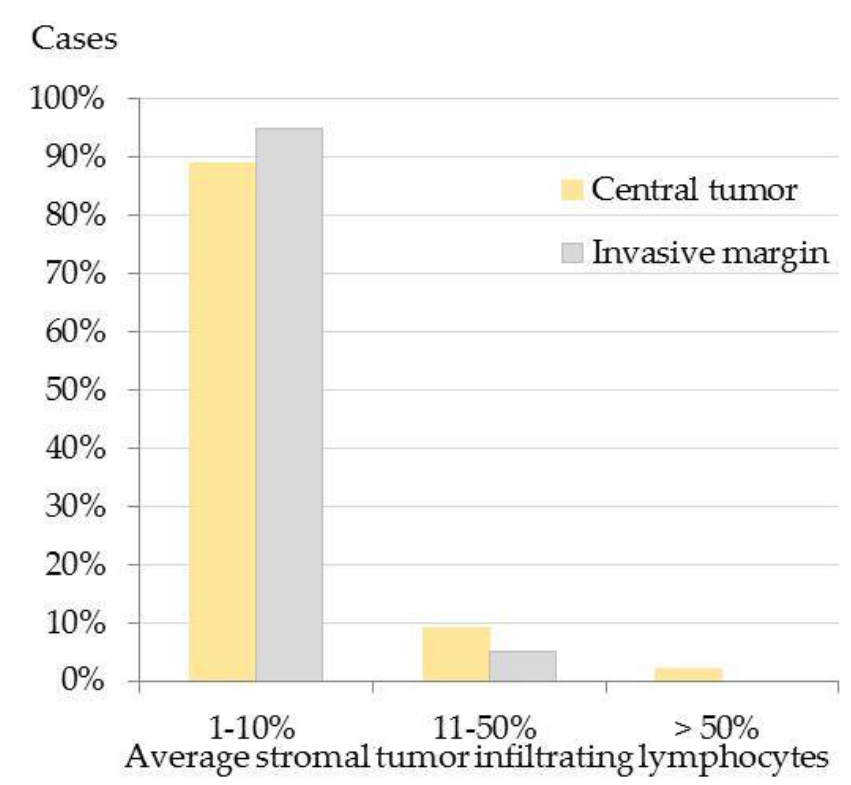

Figure 1. Average stromal tumor infiltrating lymphocytes (TILS) in the central tumor (CT) and at the infiltrative margin (IM) of pet rabbit mammary carcinomas. Most tumors encompassed $1-10 \%$ average stromal TILs in both tumor areas, i.e. 89\% (95/ 107) in the CT and 95\% (97/102) at the IM. In comparison, $11-50 \%$ average stromal TILs were detected in $9 \%(10 / 107)$ of the tumors in the CT and $5 \%(5 / 102)$ at the IM. More than 50\% TILs were only diagnosed in $2 \%(2 / 107)$ of the tumors in the CT. ${ }^{[32]}$

Stromal TILs often displayed spatial aggregations within the stroma (Figure 2), that was slightly more prominent within the CT than at the IM. ${ }^{[32]}$ At the IM, TILs were mostly located in the stroma adjacent to 
invasive tumor cell nests. ${ }^{[32]}$ The spatial clustering, that was verified by the calculation of the difference of maximal and minimal percentages of stromal TILs per $20 x$ objective FOV $[\underline{32]}$ is visualized in Figure 3.
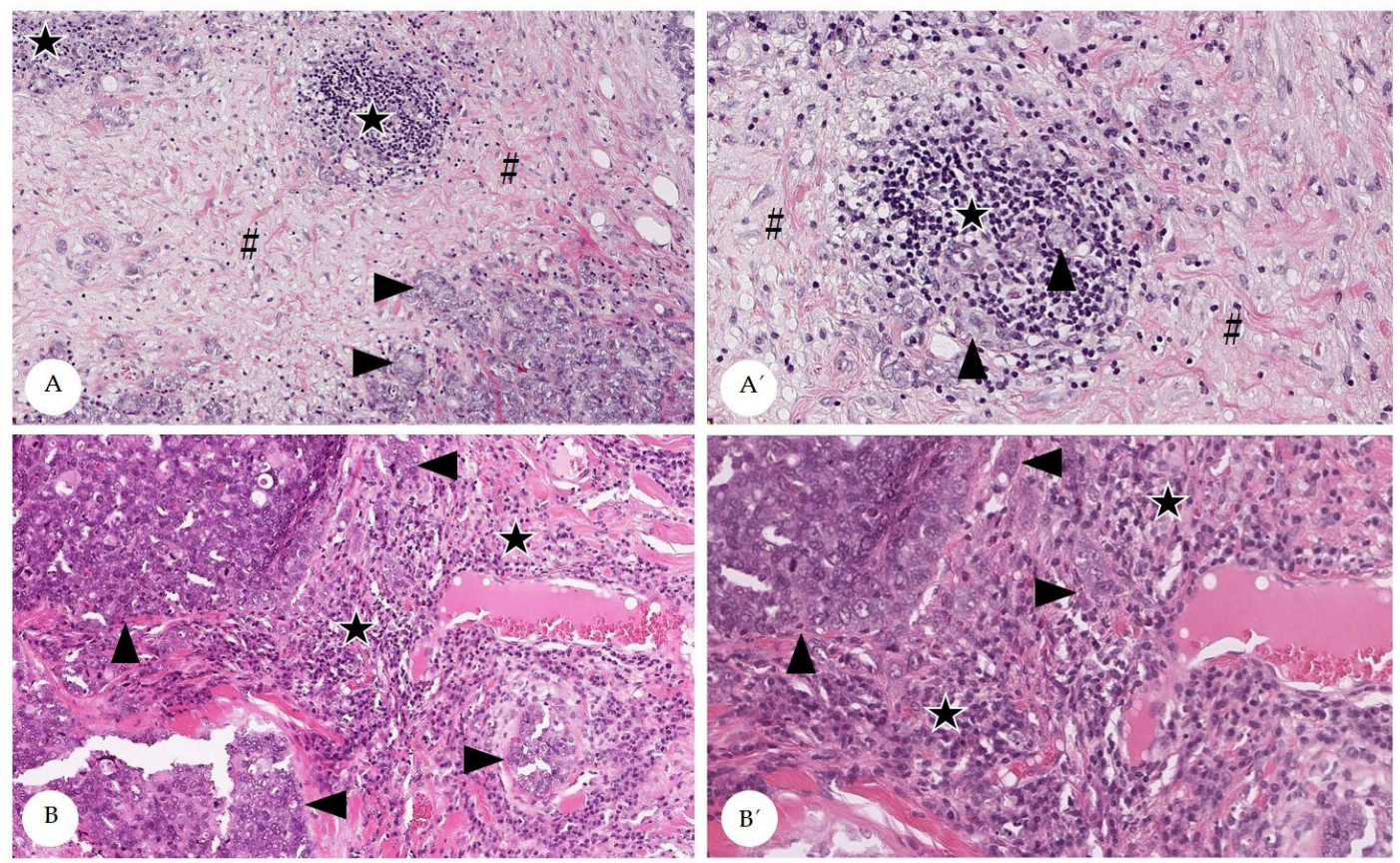

Figure 2. Spatial distribution of stromal tumor infiltrating lymphocytes (TILS) in pet rabbit mammary carcinomas. Depicted are representative examples for a heterogenous $(A)$ and a homogenous distribution (B) of stromal TILs. $A^{\prime}$ and $B^{\prime}$ depict areas from $A$ and $B$ in higher magnification. Heterogenous distribution of stromal TILs is attributed to the formation of hot spots $\left(A, A^{\prime}\right)$, whereas homogenously distributed TILs diffusely occupy the stroma ( $\left.B, B^{\prime}\right)$. Stromal areas with TILs are labelled by stars, those without TILs by hash keys. Tumor cell nests are marked by arrowheads.

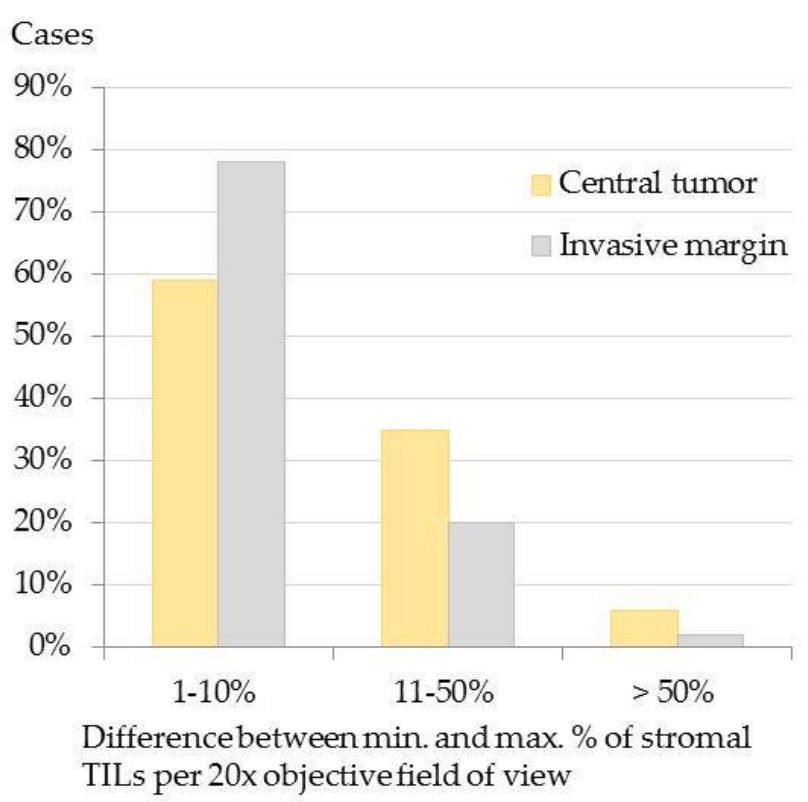

Figure 3. Spatial distribution of stromal tumor infiltrating lymphocytes (TILs) in the central tumor (CT) and at the infiltrative margin (IM) of pet rabbit mammary carcinomas. To measure the spatial distribution of stromal TILs, the difference between the maximal (max.) and minimal (min.) percentages of stromal TILs per 20x objective field of view was calculated. The difference was $1-10 \%$ in most carcinomas (CT: 59\%, 63/107; IM: 78\%, 80/102), whereas it encompassed 11-50\% in 35\% (37/107) of the carcinomas at the CT and in 20\% (20/102) at the IM. It measured > 50\% in a few carcinomas (CT: 6\%, 7/107; IM:2\%, 2/102). [32] 
The statistically significant associations for stromal TILs in the CT and IM as well as stromal TILs and the examined histological and immunohistochemical parameters are visualized in Figure 4 and are described below. For both examined tumor regions (CT and IM), average stromal TILs and maximal percentages of stromal TILs per $20 x$ objective FOV showed a statistically significant correlation. ${ }^{[32]}$ A statistically significant correlation also existed between average stromal TILs in the CT and at the IM. $\underline{\text { [32] }}$

Moreover, in the CT an increased maximal percentage of stromal TILs per 20x objective FOV was statistically associated with histological parameters suggestive of a better tumor differentiation, i.e. a reduced mitotic rate, a lower tumor grade and an increased percentage of calponin positive tumor cells. The latter was also significantly correlated with higher average percentages of stromal TILs in the CT and at the IM. [32]

An additional statistically significant correlation was obtained between a higher number of calponin positive tumor cells and a reduced mitotic rate. ${ }^{[8]}[\underline{32]}$ No statistically significant association was detected between the expression of ER $\alpha$ and PR and the numbers of stromal TILs. $\underline{\text { [32] }}$

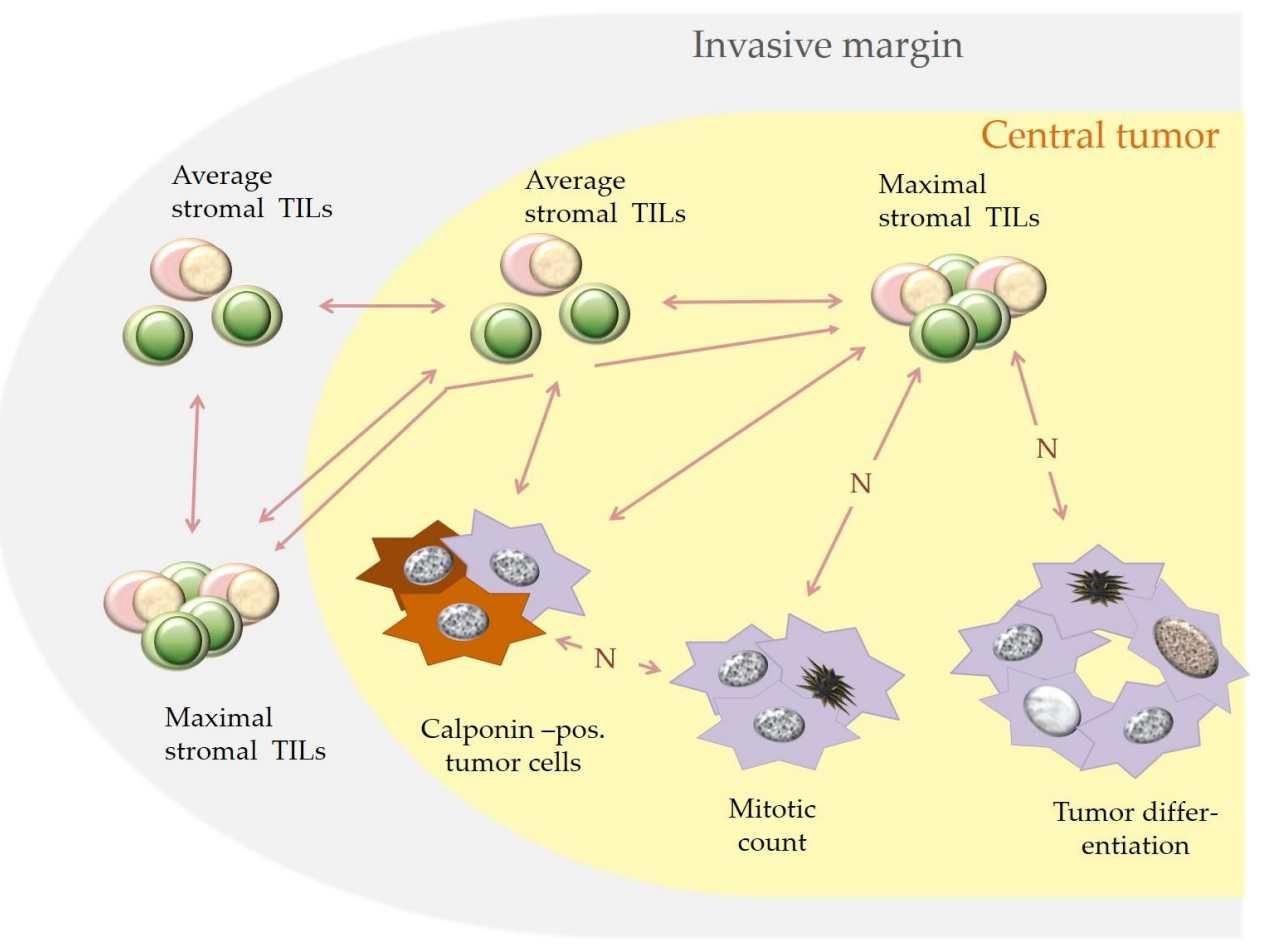

Figure 4. Depicted are statistically significant associations (bilateral arrows) that were detected in pet rabbit mammary carcinomas between increased stromal TILs and specific histological and immunohistological parameters. A negative correlation is labelled with "N".

\section{Discussion}

Results of the study of Schöniger et al $\frac{[32]}{}$ confirm that the guidelines for TIL evaluation on human tumors $[\underline{28][29]}$ can be applied to rabbit mammary carcinomas as well.

In addition, they suggest that a higher percentage of maximal stromal TILs per 20x objective FOV may be an indicator of a more favorable biological behavior in pet rabbit mammary carcinomas. This hypothesis is based on the association of higher percentage of maximal stromal TILs per 20x objective FOV with a lower mitotic count, a better tumor differentiation and a higher percentage of calponin positive tumor cells as well as an additional correlation between a higher percentage of calponin positive tumor cells and an increase in average stromal TILs. [32]

Calponin is regarded as tumor suppressor protein. $[33][34]$ By binding to the actin cytoskeleton and by interacting with signal transduction pathways, it promotes cell adhesion, reduces cellular motility, and inhibits cell division. [33][34] In rabbit mammary carcinomas, a likely favorable impact of calponin 
expression in tumor cells is further supported by the findings that a higher percentage of calponin positive tumor cells was significantly related to a lower mitotic count ${ }^{[\underline{8}][\underline{32}]}$ and a better tumor differentiation. $[\underline{8}]$

This leads to the hypothesis that also in rabbit mammary carcinomas the tumor microenvironment influences the biological behavior of the tumor. In rabbit mammary carcinomas TIL hot spot areas likely contribute to sculpturing the anti-tumor immune response. In human cancers, not only average percentages of stromal TILs, ${ }^{[28]}[\underline{29}]$ but also their spatial arrangement can have prognostic relevance and thus may assist in patient stratification. $\underline{35}][\underline{36}]$

The contents of this topical review are summarized in Figure 5.

Tumor infiltrating lymphocytes (TILs) in pet rabbit mammary carcinomas

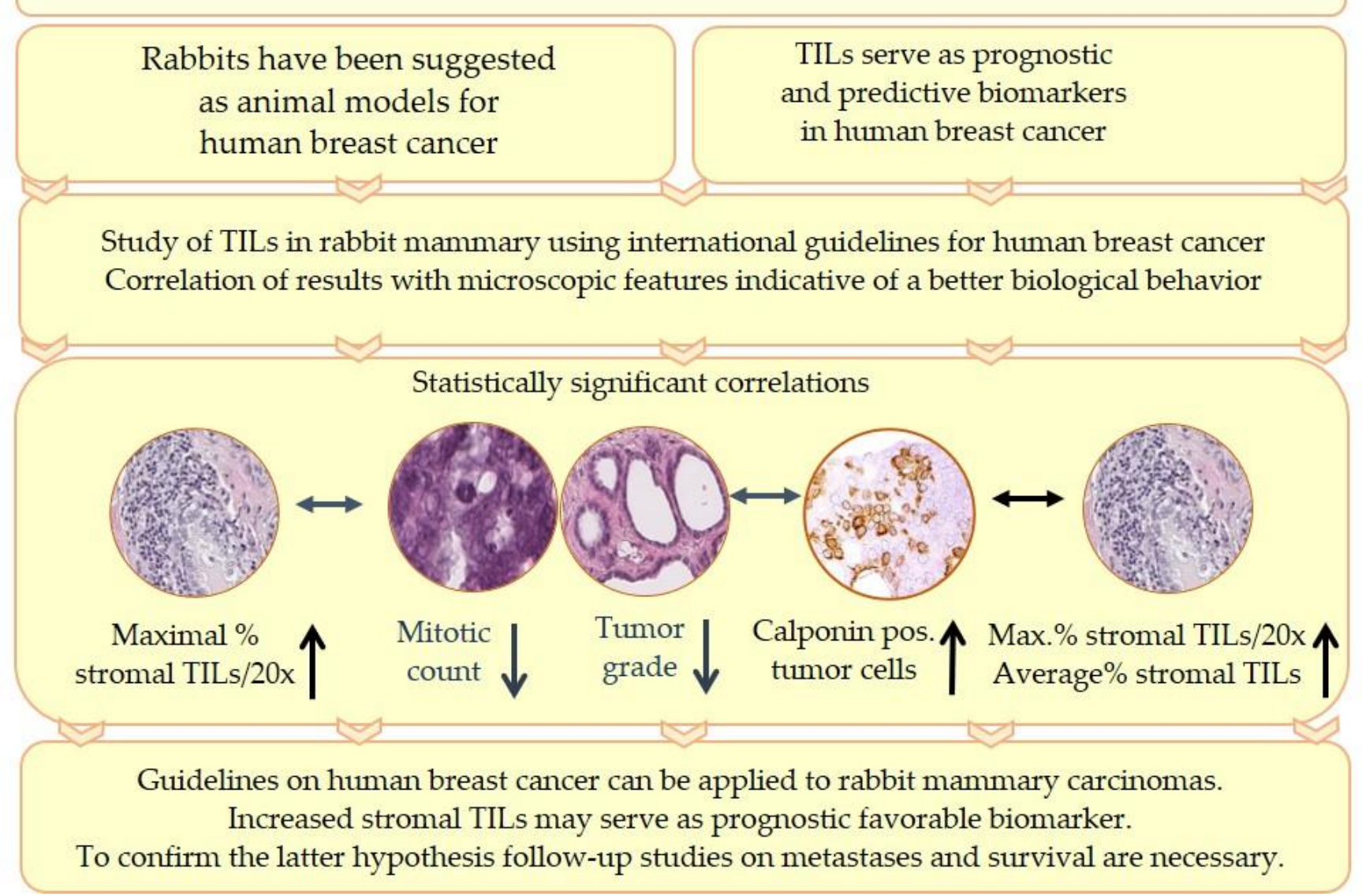

\section{Future considerations}

Future research on pet rabbit mammary carcinomas should examine the interplay of tumor associated immune cells and cancer cells. Results of these studies will likely reveal insights into cellular and molecular aspects of immunoediting also in pet rabbit mammary carcinomas. The comparison of these data with breast cancer in humans and mammary carcinomas in other species will contribute to comparative pathology and translational research into cancer biology and immune based treatment options.

The article refers to the following publication: Schöniger S, Degner S, Zhang Q, Schandelmaier C, Aupperle-Lellbach $\mathrm{H}$, Jasani B, Schoon $\mathrm{H}-\mathrm{A}$; Tumor infiltrating lymphocytes in pet rabbit mammary carcinomas: a study with relevance to comparative pathology. Animals 2020, 10,

$1437,10.3390 /$ ani10081437

\section{References}

1. DeMello M. Rabbits multiplying like rabbits. The rise in the worldwide popularity of rabbits as pets. In: Companion animals in everyday life. Situating animal-human engagement within cultures; Pregowski PM, Eds.; Palgrave 
MacMillan, Springer Nature: New York, USA, 2016; pp. 91-108.

2. Barthold SW, Griffey SM, Percy DH . Pathology of laboratory rodents and rabbits. 4th edition; Barthold SW, Griffey SM, Percy DH , Eds.; John Wiley \& Sons: Chinchester, UK, 2016; pp. 253-323.

3. Schöniger S, Degner S, Jasani B, Schoon H-A; A review on mammary tumors in rabbits: translation of pathology into medical care. Animals 2019, 9, 762, 10.3390/ani9100762.

4. Makki J; Diversity of breast carcinoma: histological subtypes and clinical relevance. Clinical Medicine Insights: Pathology 2015, 8, 23-31, 10.4137/cpath.s31563.

5. Kamińska M, Ciszewski T, Łopacka-Szatan K, Miotła P, Starosławska E; Breast cancer risk factors. Menopausal Review 2015, 3, 196-202, 10.5114/pm.2015.54346.

6. Hawsawi JM, Al-Numair NS, Sobahy TM, Al-Ajmi AM, Al-Harbi RM, Baghdadi MA, Oyouni A, Alamer OM; The role of BRCA1/2 in hereditary and familial breast and ovarian cancers. Molecular Genetics \& Genomic Medicine 2019, 7, e879, 10.1002/mgg3.879.

7. Degner S, Schoon H-A, Laik-Schandelmaier C, Aupperle-Lellbach H, Schöniger S; Estrogen receptor- $\alpha$ and progesterone receptor expression in mammary proliferative lesions of female pet rabbits. Veterinary Pathology 2018, 55, 838-848, 10.1177/0300985818788611.

8. Degner S, Schoon H-A, Degner S, Baudis M, Schandelmaier C, Aupperle-Lellbach H, Schöniger S; Expression of myoepithelial markers in mammary carcinomas of 119 pet rabbits. Animals 2019, 9, 740, 10.3390/ani9100740.

9. Schöniger S, Schoon H-A, Horn L-C; Tumors and tumor-like lesions in the mammary gland of 24 pet rabbits. Veterinary Pathology 2013, 51, 569-580, 10.1177/0300985813497486.

10. Baum B, Hewicker-Trautwein M; Classification and epidemiology of mammary tumours in pet rabbits (Oryctolagus cuniculus). Journal of Comparative Pathology 2015, 152, 291-298, 10.1016/j.jcpa.2015.01.009.

11. Elston CW, Ellis I; Pathological prognostic factors in breast cancer. I. The value of histological grade in breast cancer: experience from a large study with long-term follow-up. Histopathology 1991, 19, 403-410, 10.1111/j.13652559.1991.tb00229.x.

12. Ellis IO, Lee AHE, Pinder SE, Rakha EA. Tumors of the breast. In: Diagnostic histopathology of tumors, 4th edition; Fletcher, CDM, Eds.; Elsevier, Saunders: Philadelphia, PA, USA, 2013; pp. 1057-1145.

13. Benz CC; Impact of aging on the biology of breast cancer. Critical Reviews in Oncology/Hematology 2008, 66, 65-74, 10.1016/j.critrevonc.2007.09.001.

14. Müller K, Schall H . Kaninchen. In: Krankheiten der Heimtiere. 8th edition; Fehr M, Sassenburg L, Zwart P, Eds.; Schlütersche Verlag: Hannover, Germany, 2014; pp. 1-56.

15. Lipman NS, Zhao ZB, Andrutis KA, Hurley RJ, Fox JG, White HJ; Prolactin-secreting pituitary adenomas with mammary dysplasia in New Zealand white rabbits. Laboratory Animal Science 1994, 44, 114-120.

16. Petraitiene R, Petraitis V, Bacher J, Das SR, Parlow AF, Walsh TJ; Cyclosporine A-induced mammary hyperplasia and hyperprolactinemia in New Zealand white rabbits. Comperative Medicine 2001, 51, 430-435.

17. Sikoski P, Trybus J, Cline JM, Muhammad FS, Eckhoff A, Tan J, Lockard M, Jolley T, Britt S, Kock ND; Cystic mammary adenocarcinoma associated with a prolactin-secreting pituitary adenoma in a New Zealand white rabbit (Oryctolagus cuniculus). Comperative Medicine 2008, 58, 297-300.

18. Vicchi FL, Becu-Villalobos D; Prolactin: the bright and the dark side. Endocrinology 2017, 158, 1556-1559, 10.1210/en.2017-00184.

19. Greene HSN; Familial mammary tumors in the rabbit. III. factors concerned in their genesis and development. Journal of Experimental Medicine 1939, 70, 167-184, 10.1084/jem.70.2.167.

20. Claus EB, Schildkraut JM, Thompson WD; The genetic attributable risk of breast and ovarian cancer. Cancer 1996, 77, 2318-2324, 10.1002/(SICI)1097-0142(19960601)77:113.0.CO;2-Z..

21. Pinheiro A, Neves F, Lemos de Matos A, Abrantes J, van der Loo W, Mage R, Esteves PJ; An overview of the lagomorph immune system and its genetic diversity. Immunogenetics 2015, 68, 83-107, 10.1007/s00251-015-0868-8.

22. Esteves, PJ, Abrantes J, Baldauf H-M, Benmohamed L, Chen Y, Christensen N, et al.; The wide utility of rabbits as models of human diseases. Experimental \& Molecular Medicine 2018, 50, 1-10, 10.1038/s12276-018-0094-1.

23. Mage RG . Immunology of lagomorphs, Chapter IV. In: Handbook of vertebrate immunology; Pastoret P-P, Griebel P, Bazin H, Govaerts A, Eds.; Academic Press: San Diego, CA, USA, 1998; pp. 223-260.

24. Shiomi M. Rabbits as model for the study of human diseases, Chapter 7. In: Rabbit Biotechnology: rabbit genomics, transgenesis, cloning and models; Houdebine LM, Fan J, Eds.; Springer Science and Business Media B.V.: Dordrecht, Netherlands, Heidelberg, Germany, New York, USA, London, UK, 2009; pp. 49-64.

25. Salvakumar R, Schmidtt A, Iftner T, Ahmed R, Wettstein FO; Regression of papillomas induced by cottontail rabbit papillomavirus is associated with infiltration of CD8+ cells and persistence of viral DNA after regression. Journal of Virology 1997, 71, 5540-5548, 10.1128/JVI.71.7.5540-5548.1997.

26. Rossmann A, Mandic R, Heinis J, Höffken H, Küssner O, Kinscherf R, Weihe E, Bette M; Intraperitoneal oxidative stress in rabbits with papillomavirus-associated head and neck cancer induces tumoricidal immune response that is 
adoptively transferable. Clinical Cancer Research 2014, 20, 4289-4301, 10.1158/1078-0432.ccr-14-0677.

27. Mittal D, Gubin MM, Schreiber RD, Smyth MJ; New insights into cancer immunoediting and its three component phases--elimination, equilibrium and escape.. Current Opinion in Immunology 2014, 27, 16-25, 10.1016/j.coi.2014.01.004.

28. Salgado R, Denkert C, Demaria S, Sirtaine N, Klauschen F, Pruneri G, Wienert S, Van den Eynden G, Baehner FL, Penault-Llorca F, et al.; The evaluation of tumor-infiltrating lymphocytes (TILs) in breast cancer: Recommendations by an International TILs Working Group. Annals of Oncology 2015, 26, 259-271, 10.1093/annonc/mdu450.

29. Hendry S, Salgado R, Gevaert T, Russell PA, John T, Thapa B, Christie M, Van De Vijver K, Estrada MV, GonzalezEricsson PI, et al.; Assessing tumor-infiltrating lymphocytes in solid tumors: A practical review for pathologists and proposal for a standardized method from the international immuno-oncology biomarkers working group: Part 1: Assessing the host immune response, TILs in invasive breast carcinoma and ductal carcinoma in situ, metastatic tumor deposits and areas for further research. Advances in Anatomic Pathology 2017, 24, 235-251, 10.1097/pap.0000000000000162.

30. Gao G, Wang Z, Qu X, Zhang Z; Prognostic value of tumor-infiltrating lymphocytes in patients with triple-negative breast cancer: a systematic review and meta-analysis. BMC Cancer 2020, 20, 179-15, 10.1186/s12885-020-6668-z.

31. Denkert C, Loibl S, Noske A, Roller M, Müller BM, Komor M, Budczies J, Darb-Esfahani S, Kronenwett R, Hanusch C, von Törne C, Weichert W, Engels K, Solbach C, Schrader I, Dietel M, von Minckwitz G; Tumor-associated lymphocytes as an independent predictor of response to neoadjuvant chemotherapy in breast cancer. Journal of Clinical Oncology 2010, 28, 105-113, 10.1200/jco.2009.23.7370.

32. Schöniger S, Degner S, Zhang Q, Schandelmaier C, Aupperle-Lellbach H, Jasani B, Schoon H-A; Tumor infiltrating lymphocytes in pet rabbit mammary carcinomas: a study with relevance to comparative pathology. Animals 2020, 10, 1437, 10.3390/ani10081437.

33. Taniguchi S; Suppression of cancer phenotypes through a multifunctional actin-binding protein, calponin, that attacks cancer cells and simultaneously protects the host from invasion. Cancer Science 2005, 96, 738-746, 10.1111/j.1349-7006.2005.00118.x. 2005.

34. Liu R, Jin J-P; Calponin isoforms CNN1, CNN2 and CNN3: Regulators for actin cytoskeleton functions in smooth muscle and non-muscle cells. Gene 2016, 585, 143-153, 10.1016/j.gene.2016.02.040.

35. Nawaz S, Heindl A, Koelble K, Yuan Y; Beyond immune density: critical role of spatial heterogeneity in estrogen receptor-negative breast cancer. Modern Pathology 2015, 28, 766-777, 10.1038/modpathol.2015.37.

36. Saltz J, Gupta R, Hou L, Kurc T, Singh P, Nguyen V, Samaras D, Shroyer KR, Zhao T, Batiste R, et al.; Spatial organization and molecular correlation of tumor-infiltrating lymphocytes using deep learning on pathology images. Cell Reports 2018, 23, 181-193.e7, 10.1016/j.celrep.2018.03.086.

\section{Keywords}

comparative pathology; histopathology; mammary carcinoma; pet rabbits; translational medicine; tumor infiltrating lymphocytes 